\title{
Classical ratchet effects in heterostructures with a lateral periodic potential
}

\author{
P. Olbrich, ${ }^{1}$ J. Karch, ${ }^{1}$ E. L. Ivchenko, ${ }^{2}$ J. Kamann,,${ }^{1}$ B. März, ${ }^{1}$ M. Fehrenbacher, ${ }^{1}$ D. Weiss, ${ }^{1}$ and S. D. Ganichev ${ }^{1, *}$ \\ ${ }^{1}$ Terahertz Center, University of Regensburg, D-93040 Regensburg, Germany \\ ${ }^{2}$ A. F. Ioffe Physico-Technical Institute, Russian Academy of Sciences, 194021 St. Petersburg, Russia \\ (Received 26 January 2011; revised manuscript received 12 March 2011; published 27 April 2011)
}

\begin{abstract}
We study terahertz radiation induced ratchet currents in low dimensional semiconductor structures with a superimposed one-dimensional lateral periodic potential. The periodic potential is produced by etching a grating into the sample surface or depositing metal stripes periodically on the sample top. Microscopically, the photocurrent generation is based on the combined action of the lateral periodic potential, verified by transport measurements, and the in-plane modulated pumping caused by the lateral superlattice. We show that a substantial part of the total current is caused by the polarization-independent Seebeck ratchet effect. In addition, polarizationdependent photocurrents occur, which we interpret in terms of their underlying microscopical mechanisms. As a result, the class of ratchet systems needs to be extended by linear and circular ratchets, sensitive to linear and circular polarizations of the driving electromagnetic force.
\end{abstract}

DOI: 10.1103/PhysRevB.83.165320

PACS number(s): 05.40.-a, 05.60.Gg, 78.67.De, 73.63.Hs

\section{INTRODUCTION}

Nonequilibrium spatially periodic noncentrosymmetric systems are able to transport particles in the absence of an average macroscopic force. The directed transport in such systems, generally known as ratchet effect, has a long history and is relevant for different fields of physics. ${ }^{1-9}$ If this effect is induced by electromagnetic radiation, it is usually referred to as photogalvanic (or sometimes photovoltaic) effect, particularly if the breaking of spatial inversion symmetry is related to the microscopic structure of the system. ${ }^{10-14}$ Blanter and Büttiker ${ }^{15}$ have shown that one of the possible realizations of a ratchet is a superlattice (SL) irradiated by light through a mask of the same period but phase shifted with respect to the SL yielding a directed current due to local electron-gas heating. Recently, we have reported an experimental realization of this idea with some modifications. ${ }^{16}$ The photocurrent has been observed in semiconductor heterostructures with a onedimensional lateral periodic potential induced by etching a noncentrosymmetric grating into the sample cap layer. Hence the in-plane modulation of the pump radiation appears not via a mask with periodic structures but due to near-field effects of terahertz $(\mathrm{THz})$ radiation propagating through the grating. This photothermal effect, called also Seebeck ratchet effect, ${ }^{1}$ is polarization independent and can be generated even at normal incidence of light.

Here, we report on the observation and study of radiation induced ratchet effects sensitive to the plane of polarization of linearly polarized light and, in the case of circularly polarized light, to the photon helicity. The theoretical analysis has enabled us to propose microscopic mechanisms of the observed circular and linear ratchet effects, and to demonstrate that they are related to the combined action of an out-of-phase periodic potential and an in-plane modulated pumping of the two-dimensional electron system (2DES). The investigation of these ratchet effects has also been performed on a new set of laterally structured samples with a better controlled asymmetry.

The paper is organized as follows. In the next section, we present the theory of ratchet effects stemming from the combined action of the lateral periodic potential and the in-plane pumping by the $\mathrm{THz}$ field modulated by the near-field diffraction. We formulate the model in terms of the classical Boltzmann equation for the electron distribution function, show the position of this model with respect to other electronic ratchets, and propose a model picture to interpret the observed photocurrents. The symmetry analysis in Sec. III is followed by solving the kinetic equation (Sec. IV) and deriving equations for the Seebeck ratchet current (Sec. V) and polarization-dependent photocurrents (Sec. VI). In Sec. VII, we describe details of the sample preparation and give a short overview of the experimental technique. The experimental results are presented and discussed in Sec. VIII. Section IX summarizes the study.

\section{MODEL}

We consider a quantum well $(\mathrm{QW})$ structure modulated by a one-dimensional periodic lateral potential $V(x)$ with the period $d: V(x+d)=V(x)$. Hereafter we use the right-handed coordinate system $x, y, z$ with the axes $x, y$ lying in the interface plane and the axis $z$ parallel to the growth direction. In addition to the static potential $V(x)$, the two-dimensional electron gas is subjected to the action of an in-plane time-dependent electric field

$$
\boldsymbol{E}(x, t)=\boldsymbol{E}_{\omega}(x) e^{-i \omega t}+\boldsymbol{E}_{\omega}^{*}(x) e^{i \omega t}
$$

with the amplitude $\boldsymbol{E}_{\omega}(x)$ modulated along the $x$ axis with the same period as the static lateral potential: $\boldsymbol{E}_{\omega}(x+d)=$ $\boldsymbol{E}_{\omega}(x)$. The electric field can be linearly polarized with

$$
\operatorname{Im}\left[E_{\omega, \alpha}(x) E_{\omega, \beta}^{*}(x)\right]=0 \quad(\alpha, \beta=x, y),
$$

or circularly polarized with $E_{\omega, y}(x)=\mp i E_{\omega, x}(x)$ for $\sigma_{+}$and $\sigma_{-}$polarization, respectively. Note that the signs in the latter equation correspond to an experimental geometry where the light propagates anti-parallel to $z$.

We will describe the ratchet effects by using the classical Boltzmann equation for the electron distribution function $f_{k}(x, t)$, namely,

$$
\left(\frac{\partial}{\partial t}+\boldsymbol{v}_{\boldsymbol{k}, x} \frac{\partial}{\partial x}+\frac{\boldsymbol{F}(x, t)}{\hbar} \frac{\partial}{\partial \boldsymbol{k}}\right) f_{\boldsymbol{k}}(x, t)+Q_{\boldsymbol{k}}=0 .
$$


Here, $\boldsymbol{k}=\left(k_{x}, k_{y}\right)$ and $\boldsymbol{v}_{\boldsymbol{k}}=\hbar \boldsymbol{k} / \mathrm{m}^{*}$ are the two-dimensional electron wave vector and velocity, $m^{*}$ is the electron effective mass, $\boldsymbol{F}(x, t)$ is the force consisting of two terms

$$
\boldsymbol{F}(x, t)=-\frac{d V(x)}{d x} \hat{\boldsymbol{e}}_{x}+e \boldsymbol{E}(x, t),
$$

$e$ is the electron charge (negative), $\hat{\boldsymbol{e}}_{x}$ is a unit vector along the $x$ axis, and $Q_{k}$ is the collision integral responsible for electron momentum and energy relaxation. Equation (1) is valid for a weak and smooth potential satisfying the conditions $|V(x)| \ll$ $\varepsilon_{e}$ and $q=2 \pi / d \ll k_{e}$, where $k_{e}$ is the typical electron wave vector and $\varepsilon_{e}=\hbar^{2} k_{e}^{2} / 2 m^{*}$ is the typical energy being much larger than the photon energy $\hbar \omega$. The quantity of central interest is the average electron current

$$
j=2 e \sum_{k} \boldsymbol{v}_{k} \bar{f}_{k},
$$

where the factor 2 takes into account the electron spin degeneracy and the bar means averaging over the spatial coordinate $x$ and time $t$. In order to get a nonvanishing directed current, the force $\boldsymbol{F}(x, t)$ should be asymmetric which means that there exists no coordinate $x_{c}$ such that $\boldsymbol{F}\left(x-x_{c}, t\right)=$ $\boldsymbol{F}\left(x_{c}-x, t\right)$. Note that both the gradient $d V(x) / d x$ and the amplitude $\boldsymbol{E}_{\omega}(x)$ can possess centers of inversion but, in an asymmetric system, these centers must not coincide.

In terms of the models reviewed and discussed in Ref. 1, the system under study is a pulsating ratchet described in terms of the distribution function and Boltzmann equation with the collision integral. It is analogous to a Brownian particle in two dimensions with coordinates $x, y$ and mass $m^{*}$, which is governed by Newton's equation of motion

$$
m^{*}(\dot{\boldsymbol{v}}+\eta \boldsymbol{v})=-\nabla V(x, y, t)+\zeta(t) .
$$

Here, the pulsating force $-\nabla V(x, y, t) \equiv \boldsymbol{F}(x, t)$ is given by Eq. (2), $\eta$ is the viscous friction coefficient and $\zeta(t)$ is a randomly fluctuating force in the form of a Gaussian white noise of zero mean. The system meets the main guiding principles of a ratchet: (i) it is periodic both in space and time, (ii) the force vanishes after averaging over space and time, (iii) the system is driven permanently out of thermal equilibrium and (iv) the force $\boldsymbol{F}(x, t)$ is asymmetric.

Two mechanisms, a polarization-independent one and a polarization-dependent one, contribute to the current (3). Here, we present a qualitative interpretation of these mechanisms based on the (static) Ohm's law

$$
\boldsymbol{j}=\frac{e^{2} \tau N_{0}}{m^{*}} \boldsymbol{E},
$$

with momentum relaxation time $\tau$ and electron density $N_{0}$, and reveal the basic physics behind the ratchet effects under study. A more detailed discussion is given in Secs. V and VI. In line with the first mechanism, the modulated light field heats the electron gas and causes a periodic modulation of the effective electron temperature $\Theta(x)$ which, in its turn, leads to a redistribution of the electron density $N(x)$ and appearance of an electric-field-induced static correction $\delta N(x) \propto\left|\boldsymbol{E}_{\omega}(x)\right|^{2}$. The polarization-independent dc current is obtained from Eq. (5) if the density $N_{0}$ and the product $e E$ get replaced by $\delta N(x)$ and $-d V(x) / d x$, respectively, and the current is averaged over $x$ as follows

$j_{x}=\frac{e \tau}{m^{*}} \overline{\left[\delta N(x)\left(-\frac{d V(x)}{d x}\right)\right]}=\mu_{e} \overline{\left(\delta N(x) \frac{d V(x)}{d x}\right)}$,

where $\mu_{e}$ is the electron mobility $|e| \tau / m^{*}$. Due to the asymmetry of the system, the average of the product $\left|\boldsymbol{E}_{\omega}(x)\right|^{2}[d V(x) / d x]$ is nonzero. In the simplest case where

$$
\begin{aligned}
\boldsymbol{E}_{\omega}(x) & =\boldsymbol{E}_{0}\left[1+h_{1} \cos \left(q x+\varphi_{E}\right)\right], \\
V(x) & =V_{1} \cos \left(q x+\varphi_{V}\right),
\end{aligned}
$$

with $h_{1}$ being real, one has

$$
\begin{aligned}
j_{x} & \propto \overline{\left|\boldsymbol{E}_{\omega}(x)\right|^{2}[d V(x) / d x]} \\
& =\left|\boldsymbol{E}_{0}\right|^{2} q V_{1} h_{1} \sin \left(\varphi_{E}-\varphi_{V}\right) .
\end{aligned}
$$

In a more general case with

$$
\begin{gathered}
\boldsymbol{E}_{\omega}(x)=\boldsymbol{E}_{0}\left[1+\sum_{n=1}^{\infty} h_{n} \cos \left(n q x+\varphi_{E, n}\right)\right], \\
V(x)=\sum_{n=1}^{\infty} V_{n} \cos \left(n q x+\varphi_{V, n}\right)
\end{gathered}
$$

the current in $x$-direction is given by

$$
j_{x} \propto\left|\boldsymbol{E}_{0}\right|^{2} \sum_{n=1}^{\infty} n q V_{n} h_{n} \sin \left(\varphi_{E, n}-\varphi_{V, n}\right) .
$$

According to a classification suggested in Ref. 1, a dc current, resulting from a periodic temperature profile induced by an ac driving force, represents the Seebeck ratchet effect. This photothermal effect has been first considered theoretically by Blanter and Büttiker ${ }^{15}$ (see also Ref. 17) for a lateral superlattice covered by a periodic mask with a shifted phase respective to the SL. These authors have shown that irradiation of the SL by light through the mask results in a directional current perpendicular to the grating due to local electron gas heating. In the present work we assume, in accordance with the modulation-doped QWs used in experiment, that $|V(x)| \ll \varepsilon_{e}$ in contrast to the opposite case discussed in Ref. 15. In the experiment described below the mask is replaced by a one-dimensional array of grooves etched into the top cap of a semiconductor heterostructure or periodically arranged metallic stripes on the sample surface. The effect of the periodic structures is two-fold: Firstly, they generate a weak one-dimensional periodic potential superimposed upon the two-dimensional electron gas and, secondly, they modulate, because of near-field diffraction, the electric field amplitude and intensity of the incident light making them spatially periodic in the interface plane $(x, y)$. The system asymmetry determined by the difference between $\varphi_{E, n}$ and $\varphi_{V, n}$ is a natural consequence of the asymmetric shape of the grooves, displayed in Fig. 1(b), or of a periodically repeated asymmetric supercell $A B C A B C$. . . where the stripe width follows a 10:6:3 ratio, as displayed in Fig. 1(c). The lateral pattern gives rise to spatial modulation of the potential $V(x)$ and the near-field amplitude $\boldsymbol{E}_{\omega}(x)$ but, because of the asymmetry of the pattern, the modulation phases $\varphi_{E, n}$ and $\varphi_{V, n}$ are, in general, different. 


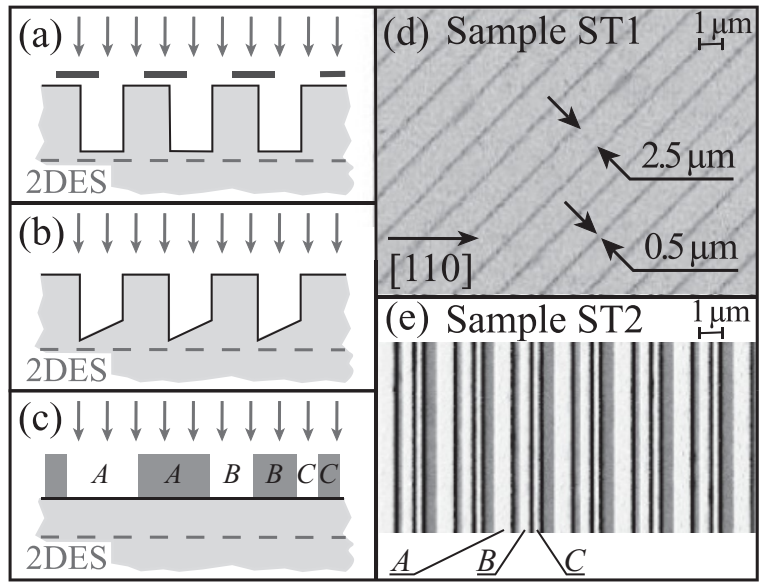

FIG. 1. Sample design. (a) Blanter and Büttiker's geometry. (b) The experimental geometry of the first set of samples (type 1, ST1) with an asymmetric groove profile. (c) The geometry of the second set of samples (type 2 , ST2) with supercells $A B C A B C \ldots$ of metallic stripes on top of the sample. (d) Electron micrograph of the first set of samples (ST1). (e) Electron micrograph of the second set of samples (ST2). Here, the widths of the patterns $A, B$, and $C$ are 1 , 0.6 , and $0.3 \mu \mathrm{m}$, respectively.

The polarization-dependent direct current stems from the time-dependent electron density oscillation $\delta N(x, t)$ linear in both the electric field $E_{x}$ and the lateral force $-d V(x) / d x$. The direct current is obtained from Eq. (5) after substituting

$$
E \rightarrow \boldsymbol{E}(x, t), \quad N_{0} \rightarrow \delta N(x, t)=\delta N_{\omega}(x) e^{-i \omega t}+\text { c.c. }
$$

and averaging over $x$ and $t$, i.e.,

$$
\begin{aligned}
\boldsymbol{j} & =\frac{e^{2} \tau}{m^{*}} \overline{[\delta N(x, t) \boldsymbol{E}(x, t)]} \\
& =2|e| \mu_{e} \operatorname{Re} \overline{\left[\delta N_{\omega}(x) \boldsymbol{E}_{\omega}^{*}(x)\right]} .
\end{aligned}
$$

For the spatial modulation defined by Eq. (7), we get again $j \propto\left|\boldsymbol{E}_{0}\right|^{2} h_{1} V_{1} \sin \left(\varphi_{E}-\varphi_{V}\right)$. Additionally, as will be shown in Sec. VI, this current also depends on the orientation of the polarization plane of linearly-polarized light and on the helicity (degree of circular polarization) in the case of circularlypolarized light. In this connection, we have supplemented the classification scheme introduced by Reimann ${ }^{1}$ by adding linear and circular ratchet effects in our previous short article. ${ }^{16}$

\section{SYMMETRY ANALYSIS}

In this section, we will analyze symmetry restrictions imposed on the polarization dependence of the ratchet currents. The system described by Eqs. (1) or (4) has the point group symmetry $C_{s}$ consisting of the identity element and the reflection $\sigma$ in the plane perpendicular to the $y$ axis. This symmetry is relevant for our asymmetric lateral superlattices. While the one-sided modulation doped QWs grown on (001)-substrates have the point group symmetry $C_{2 v}$, the adding of the asymmetric lateral potential reduces the overall symmetry to $C_{s}$ or even to $C_{1}$. It follows then that the current density components $j_{x}, j_{y}$ are related to components of the polarization unit vector $\boldsymbol{e}=\boldsymbol{E}_{0} /\left|\boldsymbol{E}_{0}\right|$ by four linearly independent coefficients

$$
\begin{gathered}
j_{x}=\bar{I}\left[\chi_{1}+\chi_{2}\left(\left|e_{x}\right|^{2}-\left|e_{y}\right|^{2}\right)\right], \\
j_{y}=\bar{I}\left[\chi_{3}\left(e_{x} e_{y}^{*}+e_{y} e_{x}^{*}\right)-\gamma P_{\text {circ }} \hat{e}_{z}\right],
\end{gathered}
$$

where $P_{\text {circ }} \hat{\boldsymbol{e}}=i\left(\boldsymbol{e} \times \boldsymbol{e}^{*}\right), \bar{I}$ is the average light intensity defined by

$$
\bar{I}=\frac{c n_{\omega}}{2 \pi}\left(\left|E_{0 x}\right|^{2}+\left|E_{0 y}\right|^{2}\right),
$$

$c$ is the light velocity in vacuum, and $n_{\omega}$ is the refractive index. Note that in the described geometry the light propagation direction and the $z$ axis are antiparallel, causing the minus sign in the second Eq. (13). The Seebeck ratchet effect is connected to the coefficient $\chi_{1}$, while the remaining three coefficients describe the linear $\left(\chi_{2}, \chi_{3}\right)$ and circular $(\gamma)$ ratchet effects.

Equations (13) should be compared to the ones of corresponding unpatterned samples, called reference samples below, or structures with a symmetric potential. One-sided modulation-doped QWs, grown along the crystallographic [001] direction of zinc-blende-lattice semiconductors have point-group symmetry $C_{2 v}$ which excludes in-plane currents for normal incidence where $E_{0 z}=0$, in contrast to the ratchet currents (13), allowed for this geometry. Under oblique incidence, the reference samples admit directional photogalvanic electric currents perpendicular to the plane of incidence, ${ }^{18,19}$

$$
\begin{aligned}
& j_{x^{\prime}}=I\left[\chi_{x^{\prime} x^{\prime} z}\left(e_{x^{\prime}} e_{z}^{*}+e_{z} e_{x^{\prime}}^{*}\right)+\gamma_{x^{\prime} y^{\prime}} P_{\text {circ }} \hat{e}_{y^{\prime}}\right], \\
& j_{y^{\prime}}=I\left[\chi_{y^{\prime} y^{\prime} z}\left(e_{y^{\prime}} e_{z}^{*}+e_{z} e_{y^{\prime}}^{*}\right)+\gamma_{y^{\prime} x^{\prime}} P_{\text {circ }} \hat{e}_{x^{\prime}}\right],
\end{aligned}
$$

which are caused by the lack of an inversion center in the reference samples at the atomic level. Here, $x^{\prime}$ and $y^{\prime}$ denote the axes [110] and [110], respectively, $\chi$ and $\boldsymbol{\gamma}$ are a third-order tensor and a second-order pseudotensor describing the linear (LPGE) and circular (CPGE) photogalvanic effects, respectively. Equations (14) show that in reference samples a photocurrent can be generated only at oblique incidence $(z$ component of the radiation electric field is needed). This is in contrast to the asymmetric lateral structures where the current given by Eqs. (13) reaches a maximum at normal incidence.

Since the lateral superstructure is responsible for the photogalvanic effects observed at normal incidence then one can, while developing a theory of the normal-incident currents, ignore the initial (microscopic) symmetry $C_{2 v}$ of the reference heterostructure, disregard mechanisms of photocurrents related to the lack of an inversion center in the unstructured sample, and rely only on the symmetry of the superstructure potential $V(x)$ and the in-plane intensity modulation. In this case Eqs. (13) can be applied for any orientation of the axes $x, y$ irrespectively to the crystallographic directions [110], [110]. At oblique incidence, the photocurrent can be naturally described by a sum of superstructure-induced and intrinsic contributions, Eqs. (13) and (14).

In the lateral structure ST1 sketched in Fig. 1(b), the grooves are oriented along the [100] direction and the axes $x\|[100], y\|$ [010] in Eqs. (13) are rotated around $z$ by $45^{\circ}$ with respect to $x^{\prime}, y^{\prime}$; in the sample ST2 of Fig. 1(c) the axes $x, y$ coincide with $x^{\prime}, y^{\prime}$. In both patterned samples ST1 and ST2, obliquely incident light generates both the ratchet current (13) and the photogalvanic current (14). This allows us to compare the 
contributions to the photocurrents due to the lack of inversion symmetry on the atomic (intrinsic mechanisms) and on the micron scale (periodic grating) experimentally.

\section{EXPANSION IN THE PERTURBATION THEORY}

The ratchet currents given by Eqs. (6) and (12) can be obtained by solving the kinetic equation (1) in third-order perturbation theory, i.e., second order in the electric-field amplitude and first order in the static lateral potential. In this work, we use the collision integral $Q_{k}$ in the convenient form of a sum of the elastic scattering term $Q_{k}^{(\text {el.sc.) }}$ and the energy relaxation term $Q_{\varepsilon}$. The former is taken in the simplest form,

$$
Q_{k}^{\text {(el.sc. })}=\frac{f_{k}(x, t)-\left\langle f_{k}(x, t)\right\rangle}{\tau},
$$

where the brackets mean the average over the directions of $\boldsymbol{k}$, and $\tau$ is the momentum scattering time assumed to be constant. The term $Q_{\varepsilon}$ is treated in the approximation of effective temperature; see below.

The electron distribution function is expanded in powers of the light electric field up to the second order,

$$
f_{\boldsymbol{k}}(\boldsymbol{\rho})=f_{\boldsymbol{k}}^{(0)}(x)+f_{\boldsymbol{k}}^{(1)}(x, t)+f_{\boldsymbol{k}}^{(2)}(x, t),
$$

where $f_{\boldsymbol{k}}^{(0)}(x)$ is the equilibrium distribution function given by

$$
f_{\boldsymbol{k}}^{(0)}(x)=\left[\exp \left(\frac{\varepsilon_{\boldsymbol{k}}+V(x)-\mu_{0}}{k_{B} T}\right)+1\right]^{-1}
$$

with $\mu_{0}, k_{B}$, and $T$ being the chemical potential, Boltzmann constant and absolute temperature, respectively, and $\varepsilon_{\boldsymbol{k}}$ being the electron energy $\hbar^{2} k^{2} / 2 m^{*}$. Here we consider the limit of high temperatures and assume that the electron gas obeys a nondegenerate statistics. Then, retaining terms of zero and first orders in the lateral potential, we can approximate the equilibrium function by

$$
f_{k}^{(0)}(x)=\left(1-\frac{V(x)}{k_{B} T}\right) \exp \left(\frac{\mu_{0}-\varepsilon_{k}}{k_{B} T}\right) .
$$

The first-order correction is time dependent and can be arrranged as a sum of two complex-conjugate monoharmonic terms,

$$
f_{\boldsymbol{k}}^{(1)}(x, t)=e^{-i \omega t} f_{\boldsymbol{k} \omega}^{(1)}(x)+e^{i \omega t} f_{\boldsymbol{k} \omega}^{(1) *}(x) .
$$

For the second-order correction, it is sufficient to retain the time-independent contribution $f_{k}^{(2)}(x) \equiv \xi_{k}(x)$ only and to reduce Eq. (3) to

$$
j=2 e \sum_{k} v_{k} \bar{\xi}_{k}
$$

By successive iteration of the kinetic equation, we obtain equations for first- and second-order corrections,

$$
\begin{gathered}
\left(-i \omega+\frac{1}{\tau}+v_{x} \frac{\partial}{\partial x}-\frac{d V(x)}{d x} \frac{1}{\hbar} \frac{\partial}{\partial k_{x}}\right) f_{\boldsymbol{k} \omega}^{(1)}(x) \\
-\frac{\left\langle f_{\boldsymbol{k} \omega}^{(1)}(x)\right\rangle}{\tau}+Q_{\varepsilon}^{(1)}=-\frac{e}{\hbar} \boldsymbol{E}_{\omega}(x) \frac{\partial}{\partial \boldsymbol{k}} f_{\boldsymbol{k}}^{(0)}(x),
\end{gathered}
$$

$$
\begin{aligned}
& \left(\frac{1}{\tau}+v_{x} \frac{\partial}{\partial x}-\frac{d V(x)}{d x} \frac{1}{\hbar} \frac{\partial}{\partial k_{x}}\right) \xi_{\boldsymbol{k}}(x)-\frac{\left\langle\xi_{\boldsymbol{k}}(x)\right\rangle}{\tau} \\
& +Q_{\varepsilon}^{(2)}=-\frac{2 e}{\hbar} \operatorname{Re} \overline{\left[\boldsymbol{E}_{\omega}^{*}(x) \frac{\partial}{\partial \boldsymbol{k}} f_{\boldsymbol{k} \omega}^{(1)}(x)\right]},
\end{aligned}
$$

where the superscript $j$ in $Q_{\varepsilon}^{(j)}$ labels the order of correction to the collision integral. Next, we multiply terms in the equation for $\xi_{\boldsymbol{k}}(x)$ by $2 e \boldsymbol{v}_{\boldsymbol{k}}$, sum over $\boldsymbol{k}$ and obtain for the ratchet current

$$
\begin{aligned}
\boldsymbol{j}= & \frac{2 e \tau}{\hbar} \sum_{\boldsymbol{k}} \boldsymbol{v}_{\boldsymbol{k}} \frac{\overline{d V(x)} \frac{\partial \xi_{\boldsymbol{k}}(x)}{\partial x}}{\partial k_{x}} \\
& -\frac{4 e^{2} \tau}{\hbar} \sum_{\boldsymbol{k}} \boldsymbol{v}_{\boldsymbol{k}} \operatorname{Re} \overline{\left[\boldsymbol{E}_{\omega}^{*}(x) \frac{\partial}{\partial \boldsymbol{k}} f_{\boldsymbol{k} \omega}^{(1)}(x)\right]} .
\end{aligned}
$$

Integrating by parts over $\boldsymbol{k}$ and introducing the spatially modulated electron densities

$$
\delta N(x)=2 \sum_{\boldsymbol{k}} \xi_{\boldsymbol{k}}(x) \quad \text { and } \quad \delta N_{\omega}(x)=2 \sum_{\boldsymbol{k}} f_{\boldsymbol{k} \omega}^{(1)}(x),
$$

we arrive at the final equation,

$$
\boldsymbol{j}=\mu_{e}\left\{\overline{\delta N(x) \frac{d V(x)}{d x}}+2|e| \operatorname{Re} \overline{\left[\boldsymbol{E}_{\omega}^{*}(x) \delta N_{\omega}(x)\right]}\right\},
$$

which is just the sum of the two currents (6) and (12) derived heuristically in Sec. II.

The further development of the theory is based on additional assumptions: (i) the energy relaxation time $\tau_{\varepsilon}$ is assumed to exceed the momentum relaxation time $\tau$; (ii) the electron mean free path $l_{e}=v_{T} \tau$ and energy diffusion length $l_{\varepsilon}=v_{T} \sqrt{\tau \tau_{\varepsilon}}$ (see, e.g., Ref. 20) are both small compared with the SL period $d$; (iii) we neglect the influence of ac diffusion on the first-order amplitudes $f_{k \omega}^{(1)}(x)$, which is valid if $v_{T} q \ll \omega$, where $v_{T}$ is the thermal velocity $\sqrt{2 k_{B} T / m^{*}}$, or, equivalently, if the period of the light, $2 \pi / \omega$, is shorter than $d / v_{T}$, the time of the free flight of an electron over the spatial period $d$. On the other hand, no restrictions are imposed on the value of the product $\omega \tau$.

\section{SEEBECK RATCHET EFFECT}

To calculate the Seebeck ratchet current (6), represented also by the first term in Eq. (22), we need to find a static correction $\delta N(x)$ of the spatially modulated electron density. Since the derivative $d V(x) / d x$ already enters the right-hand side of Eq. (6), this correction can be found neglecting the lateral potential. In this case, we can approximately replace the inhomogeneous term in Eq. (20) by

$$
\begin{aligned}
g\left(\varepsilon_{\boldsymbol{k}}, x\right) & \equiv-\frac{2 e}{\hbar} \operatorname{Re}\left[\boldsymbol{E}_{\omega}^{*}(x)\left\langle\frac{\partial}{\partial \boldsymbol{k}} f_{\boldsymbol{k} \omega}^{(1)}(x)\right\rangle\right] \\
& =\frac{2 e^{2} \tau\left|\boldsymbol{E}_{\omega}(x)\right|^{2}}{m^{*}\left(1+\omega^{2} \tau^{2}\right)} \frac{\varepsilon_{\boldsymbol{k}}-k_{B} T}{\left(k_{B} T\right)^{2}} f_{\boldsymbol{k}}^{(0)},
\end{aligned}
$$

where $f_{k}^{(0)}=\exp \left[\left(\mu_{0}-\varepsilon_{k}\right) / k_{B} T\right]$ is the equilibrium distribution function normalized to the average electron density $N_{0}$. Equation (20) with the inhomogeneous term $g\left(\varepsilon_{\boldsymbol{k}}, x\right)$ can be reduced to the following macroscopic equations for the two-dimensional electron density $N(x)$, local nonequilibrium 
temperature $\Theta(x)$, current density $j_{x}$, and energy flux density $\mathcal{J}(x)$ :

$$
\begin{gathered}
j_{x}=\mu_{e}\left\{N(x) \frac{d V(x)}{d x}+\frac{d}{d x}\left[k_{B} \Theta(x) N(x)\right]\right\}, \\
\frac{d j_{x}}{d x}=0, \\
\mathcal{J}=\left[2 k_{B} \Theta(x)+V(x)\right] \frac{j_{x}}{e} \\
-\frac{2 \mu_{e}}{|e|} N(x) k_{B}^{2} \Theta(x) \frac{d \Theta(x)}{d x}, \\
\frac{d \mathcal{J}}{d x}=\hbar \omega G(x) N(x)-\frac{k_{B}[\Theta(x)-T]}{\tau_{\varepsilon}} N(x) .
\end{gathered}
$$

Here, we introduced the energy relaxation time $\tau_{\varepsilon}$ and the generation rate $G(x)$ defined as the Drude absorption rate per particle,

$$
\begin{aligned}
G(x) & =\frac{2 \sum_{\boldsymbol{k}} \varepsilon_{\boldsymbol{k}} g\left(\varepsilon_{\boldsymbol{k}}, x\right)}{\hbar \omega N_{0}}=\frac{2 e^{2} \tau\left|\boldsymbol{E}_{\omega}(x)\right|^{2}}{m^{*} \hbar \omega\left(1+\omega^{2} \tau^{2}\right)} \\
& =\frac{4 \pi e^{2}}{m^{*} c n_{\omega}} \frac{\tau}{1+\omega^{2} \tau^{2}} \frac{I(x)}{\hbar \omega} .
\end{aligned}
$$

For the sake of completeness, we deliberately included into the set of Eqs. (23) terms originating in the lateral potential. One can check that averaging of Eq. (23a) over $x$ leads to Eq. (6).

Under homogeneous optical excitation, $G(x) \equiv G_{0}$, Eqs. (23) have the following solution:

$$
k_{B} \Theta=k_{B} T+\hbar \omega G_{0} \tau_{\varepsilon}, \quad N(x)=N_{0} e^{-V(x) / k_{B} \Theta},
$$

where $N_{0}$ is $x$ independent. For this solution, both $j_{x}$ and $\mathcal{J}$ vanish. The current $j_{x}$ becomes nonzero only if the generation rate $G$ varies spatially. For the simple spatial modulation (7) of the electric field with a small coefficient $h_{1}$, we write $G(x)=G_{0}\left[1+2 h_{1} \cos \left(q x+\varphi_{E}\right)\right]$. Neglecting the energy diffusion term in Eqs. (23) we obtain that the steady-state generation produces a stationary periodic electron temperature $\Theta(x)$ with $\Theta(x)-\bar{\Theta} \equiv \delta \Theta(x)=k_{B}^{-1} \tau_{\varepsilon} \hbar \omega\left[G(x)-G_{0}\right]$. Now it follows from Eq. (23a) that this temperature modulation is accompanied by a light-induced periodic correction to the electron density $\delta N(x) \approx-N_{0} \delta \Theta(x) / \bar{\Theta}$.

For the lateral potential given by Eq. (7) where the symmetry of the system is broken by a phase shift between $V(x)$ and $\Theta(x)$, the final result reads

$$
\begin{aligned}
j_{x} & =\chi_{1}^{S} \bar{I}=\zeta \mu_{e} N_{0} \hbar q \omega \tau_{\varepsilon} \frac{G_{0} V_{1}}{2 k_{B} T} \\
& =\zeta \frac{4 \pi e^{2}}{\hbar c n_{\omega}} \frac{\hbar q}{m^{*}} \frac{\mu_{e} N_{0} \tau \tau_{\varepsilon}}{1+\omega^{2} \tau^{2}} \frac{\bar{I} V_{1}}{k_{B} T},
\end{aligned}
$$

where $\bar{I}$ is the averaged light intensity, $\zeta=h_{1} \sin \left(\varphi_{V}-\varphi_{E}\right)$ is the asymmetry parameter related to the inhomogeneous photoexcitation, and $\bar{\Theta}$ is replaced by $T$. The Seebeck ratchet current (24) is polarization independent and increases with decreasing temperature. For a more complicated spatial modulation (9), the product $\zeta q V_{1}$ should be replaced by $\sum_{n} n q V_{n} h_{n} \sin \left(\varphi_{V, n}-\varphi_{E, n}\right)$.

\section{POLARIZATION-DEPENDENT RATCHET CURRENTS}

Now we turn to the polarization dependent mechanisms of the currents and discuss the linear and circular ratchet effects described in Eqs. (13) by the terms proportional to $\chi_{2}, \chi_{3}$, and $\gamma$. We will show that these ratchet currents can also be generated in a lateral SL with the out-of-phase periodic potential $V(x)$ and electric field $\boldsymbol{E}_{\omega}(x)$. For this purpose we consider the second term in Eq. (22) or, equivalently, the contribution (12). The oscillation $\delta N_{\omega}(x)$ entering Eqs. (12), (22) and defined by Eq. (21) satisfies the continuity equation

$$
-i \omega \delta N_{\omega}(x)+\frac{\partial j_{\omega, x}(x)}{\partial x}=0,
$$

where $j_{\omega, x}(x)$ is the amplitude of current oscillations at frequency $\omega$. It follows from Eq. (25) that, in order to calculate the current given by Eq. (12), it is sufficient to find a contribution to $\delta N_{\omega}(x)$, linear in the lateral potential and a nonmodulated electric field replacing $\boldsymbol{E}_{\omega}(x)$ by $\boldsymbol{E}_{0}$ in Eq. (19).

The function $f_{\boldsymbol{k} \omega}^{(1)}(x)$ is conveniently rewritten as

$$
f_{\boldsymbol{k} \omega}^{(1)}(x)=\frac{e \boldsymbol{E}_{0} \boldsymbol{v}_{\boldsymbol{k}} \tau_{\omega}}{k_{B} T} f_{\boldsymbol{k}}^{(0)}(x)+F_{\boldsymbol{k} \omega}(x),
$$

where $\tau_{\omega}=\tau /(1-i \omega \tau)$. The correction $F_{k \omega}(x)$ should be calculated in first order in the lateral potential and, in this approximation, satisfies the equation

$$
\begin{gathered}
\left(-i \omega+\frac{1}{\tau}+v_{x} \frac{\partial}{\partial x}\right) F_{\boldsymbol{k} \omega}(x)-\frac{\left\langle F_{\boldsymbol{k} \omega}(x)\right\rangle}{\tau}+Q_{\varepsilon}^{(1)} \\
=\frac{d V(x)}{d x} \frac{e E_{0 x} \tau_{\omega}}{m^{*} k_{B} T} f_{\boldsymbol{k}}^{(0)} .
\end{gathered}
$$

On summing this equation over $\boldsymbol{k}$ and neglecting the ac diffusion, we find

$$
\delta N_{\omega}(x)=\frac{i e \tau_{\omega} N_{0}}{\omega m^{*} k_{B} T} \frac{d V(x)}{d x} E_{0 x} .
$$

Substituting Eqs. (28) to (12) and averaging over $x$ we obtain the ratchet photocurrents, which, together with the polarization independent current (24), can be written as

$$
\begin{gathered}
j_{x}=\bar{I}\left[\chi_{1}+\chi_{2}\left(\left|e_{x}\right|^{2}-\left|e_{y}\right|^{2}\right)\right], \\
j_{y}=\bar{I}\left[\chi_{3}\left(e_{x} e_{y}^{*}+e_{y} e_{x}^{*}\right)-\gamma P_{\text {circ }} \hat{e}_{z}\right],
\end{gathered}
$$

with coefficients

$$
\chi_{2}=\chi_{3}=-\omega \tau \gamma, \quad \chi_{1}=\chi_{1}^{S}-\omega \tau \gamma
$$

and

$$
\gamma=\zeta \frac{\pi e^{2}}{\hbar c n_{\omega}} \frac{\hbar q}{m^{*}} \frac{V_{1}}{k_{B} T} \frac{\mu_{e} N_{0} \tau}{\omega\left(1+\omega^{2} \tau^{2}\right)},
$$

being in full agreement with the phenomenological Eqs. (13). It should be noted that so far the parameter $\tau$ was kept constant. In the general case of energy-dependent momentum relaxation time $\tau(\varepsilon)$, the ratchet currents acquire an additional contribution proportional to $d \ln \tau(\varepsilon) / d \ln \varepsilon$; see e.g., Refs. 21 and 22. We also note that we used the condition $\tau_{\varepsilon} \ll \tau$ while deriving the equations for the coefficients $\gamma$ and $\chi_{j}$. If these relaxation time parameters are comparable, the relation between the phenomenological coefficients can change but Eqs. (24) and (31) can still be used to estimate the order of magnitude of the currents. 
The allowance for polarization-dependent effects is a fundamental difference between systems with one and more than one dimensional character of motion. Even if the pulsating force is modulated in one dimension, say, along the axis $x$, as presented by Eq. (2), but the carriers can move in two dimensions $x$ and $y$, both components of the electric field, $E_{\omega, x}$ and $E_{\omega, y}$, act on the electron motion. In this case the ratchet current is related not only to the squared modulus $\left|E_{\omega, x}\right|^{2}$ as in the one-dimensional ratchet with the particle's motion along one axis $x$, but it also contains contributions proportional to $\left|E_{\omega, y}\right|^{2}$ as well as to real and imaginary parts of the product $E_{\omega, x} E_{\omega, y}^{*}$. The density oscillation (28) is caused by the $x$ component of the electric field but, according to Eq. (12), both components of the electric field act on this oscillation resulting in a ratchet current both in $x$ ("longitudinal ratchet effect") and $y$ ("transverse ratchet effect") directions. Moreover, the current $j_{y}$ depends on the difference between phases of the complex amplitudes $E_{0 x}$ and $E_{0 y}$. If the phases coincide, the light is linearly polarized and gives rise to the linear ratchet current proportional to the coefficient $\chi_{3}$ in Eq. (13). If the phases differ by $\pm 90^{\circ}$, the light is circularly polarized and induces the circular ratchet current described by the coefficient $\gamma$.

\section{SAMPLES AND EXPERIMENTAL METHODS}

We study photocurrents in GaAs/AlGaAs heterostructures employing two types of lateral superlattice gratings.

The first type of superlattice (ST1) consists of asymmetrically etched grooves with a SL period $d$ of $2.5 \mu \mathrm{m}$. A corresponding sketch of the grating and an electronic micrograph are shown in Figs. 1(b) and 1(d), respectively. The superlattices were prepared on molecular-beam epitaxy (001)grown $\mathrm{Si}-\delta$-doped $n$-type $\mathrm{GaAs} / \mathrm{Al}_{0.25} \mathrm{Ga}_{0.75} \mathrm{As} \mathrm{QW}$ structures having at $T=4.2 \mathrm{~K}(=300 \mathrm{~K})$ a mobility $\mu_{e} \approx 4.8 \times$ $10^{6} \mathrm{~cm}^{2} / \mathrm{Vs}\left(\approx 6 \times 10^{3} \mathrm{~cm}^{2} / \mathrm{Vs}\right)$, and a carrier density $N_{0}$ of $2 \times 10^{11} \mathrm{~cm}^{-2}\left(\approx 1.2 \times 10^{11} \mathrm{~cm}^{-2}\right)$. At room temperature the electron mean free path $l_{e}$ is $0.3 \mu \mathrm{m}$ and hence the condition $l_{e} \ll d$ holds. For the experiments we used $5 \mathrm{~mm} \times 5 \mathrm{~mm}$ square shaped samples oriented along the [1ㅣㅣㄹ and [110] directions. To measure photocurrents, pairs of ohmic contacts were alloyed in the middle of each sample edge. Grooves with $0.5 \mu \mathrm{m}$ width and a period of $2.5 \mu \mathrm{m}$ were obtained by electron-beam lithography and subsequent reactive ion etching using $\mathrm{SiCl}_{4}$. Care was taken not to etch through the twodimensional electron system. In order to get a large patterned area of about $1.4 \mathrm{~mm}^{2}$, 64 squares, each $150 \mu \mathrm{m} \times 150 \mu \mathrm{m}$, were stitched together. In sample ST1 the one-dimensional grating is oriented along the [100] cubic direction, with a slight misalignment of about $4^{\circ}$. The micrographs reveal an asymmetric shape of the grooves: The average depth on the right side of a groove is smaller than that on the left side. The reason for this is ascribed to the different etching velocities along the [110] and [110] directions. ${ }^{23,24}$ As reference samples for this set of structures we used unpatterned samples R1 and/or structures R2 with grooves very close to $\langle 110\rangle$. The cross section of these grooves is oriented rather symmetrically and does not introduce a structure asymmetry.

To achieve a better control of the asymmetry (which in the previous set of samples depends on anisotropic etching) and to enable both transport and photocurrent measurements in one and the same device, another set of samples, ST2, has been fabricated. For these devices, the lateral superlattices were prepared on a (001)-grown $\mathrm{Si}-\delta$-doped $\mathrm{GaAs} / \mathrm{Al}_{0.28} \mathrm{Ga}_{0.72} \mathrm{As}$ heterostructure. The room-temperature mobility and carrier density in the structures without grating are $\mu_{e}=3.2 \times$ $10^{3} \mathrm{~cm}^{2} / \mathrm{Vs}$ and $N_{0}=1.8 \times 10^{12} \mathrm{~cm}^{-2}$. In order to compare the data of modulated and unmodulated two-dimensional electron systems, we prepared a Hall bar geometry with a patterned region and an unpatterned reference part, as shown in Fig. 7. The SL is defined by $e$-beam lithography and deposition of micropatterned gate fingers using $15 \mathrm{~nm}$ Ti and $120 \mathrm{~nm}$ $\mathrm{Au}$. The schematics of the gate fingers, consisting of stripes having three different widths $A=1 \mu \mathrm{m}, B=0.6 \mu \mathrm{m}$, and $C=0.3 \mu \mathrm{m}$ with ratio $A: B: C=10: 6: 3$, and separated by $A, B$, and $C$, is shown in Fig. 1(c) and a corresponding electron micrograph in Fig. 1(e). This asymmetric supercell is repeated to generate an asymmetric but periodic potential superimposed upon the two-dimensional electron system. The asymmetric supercells $A B C A B C \ldots$ are patterned on a $500 \times 140 \mu \mathrm{m}^{2}$ area and generate a strain-induced potential in the 2DES with a period $d$ of $3.8 \mu \mathrm{m}$. The gate fingers, which are all connected and grounded, are oriented along the $\langle 110\rangle$ direction, perpendicularly to the Hall bar. Both parts of the Hall bar were characterized by magnetotransport measurements in a top-loading $\mathrm{He}^{3} / \mathrm{He}^{4}$ dilution cryostat at $100 \mathrm{mK}$ using standard four-probe lock-in technique. To avoid heating of free charge carriers small currents, which do not exceed $100 \mathrm{nA}$, have been applied. The resulting lowtemperature electron density (mobility) are $N_{0}=2.3 \times$ $10^{11} \mathrm{~cm}^{-2}\left(\mu_{e}=1.1 \times 10^{6} \mathrm{~cm}^{2} / \mathrm{Vs}\right)$ in the modulated and $N_{0}=2.3 \times 10^{11} \mathrm{~cm}^{-2}\left(\mu_{e}=1.5 \times 10^{6} \mathrm{~cm}^{2} / \mathrm{Vs}\right)$ in the unmodulated 2DES.

For optical excitation we used a pulsed molecular $\mathrm{THz}$ laser with $\mathrm{NH}_{3}$ as an active medium. ${ }^{25,26}$ Circularly and linearly polarized radiation pulses of about $100 \mathrm{~ns}$ duration with the wavelength $\lambda=280 \mu \mathrm{m}$ and power $P \simeq 2 \mathrm{~kW}$ were applied. The photocurrents were induced by indirect intrasubband (Drude-like) optical transitions in the lowest size-quantized subband. Various polarization states of the radiation are achieved by transmitting the linearly polarized $(\boldsymbol{E} \| y$ ) laser beam through $\lambda / 2$ or $\lambda / 4$ crystal quartz plates. By rotating the $\lambda / 4$ plate, one transfers the linear into elliptical polarization. The polarization states are directly related to the angle $\varphi$ between the initial linear polarization of the laser light and the optical axis of the plate, resulting in $P_{\text {circ }}=\sin 2 \varphi$ for the degree of circular polarization and for the bilinear combinations of the polarization vector components in Eqs. (13),

$$
\begin{aligned}
& \mathcal{S}(\varphi) \equiv e_{x} e_{y}^{*}+e_{y} e_{x}^{*}=-\frac{1}{2} \sin 4 \varphi, \\
& \mathcal{C}(\varphi) \equiv\left|e_{x}\right|^{2}-\left|e_{y}\right|^{2}=-\cos ^{2} 2 \varphi .
\end{aligned}
$$

If the plane of polarization of linearly polarized light incident upon a $\lambda / 2$ plate is at an angle $\varphi_{\lambda / 2}$ with respect to the slow axis the plane of polarization of the transmitted light is rotated by an angle $\alpha=2 \varphi_{\lambda / 2}$ and the above bilinear combinations are given by

$$
\mathcal{S}(\alpha)=-\sin 2 \alpha, \quad \mathcal{C}(\alpha)=-\cos 2 \alpha
$$




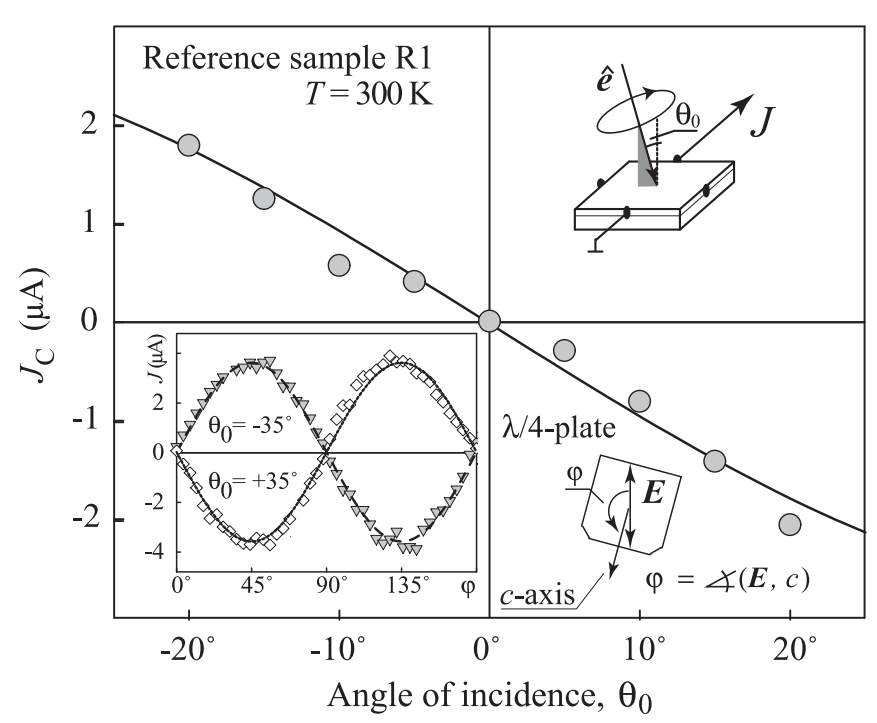

FIG. 2. Circular photogalvanic current $J_{C}=\left[J\left(\varphi=45^{\circ}\right)-\right.$ $\left.J\left(\varphi=135^{\circ}\right)\right] / 2$ measured as a function of the angle of incidence $\theta_{0}$ in a (001)-oriented $\mathrm{GaAs} / \mathrm{Al}_{0.25} \mathrm{Ga}_{0.75}$ reference $\mathrm{QW}$ sample $\mathrm{R} 1$ without a lateral structure. The current is measured at room temperature in the direction normal to the light propagation. The photocurrent is excited by radiation with the wavelength $\lambda=280 \mu \mathrm{m}$ and power $P \approx 2 \mathrm{~kW}$. The inset (bottom, left) shows the dependence of the total photocurrent $J$ on the angle $\varphi$ measured for angles of incidence $\theta_{0}= \pm 35^{\circ}$. Two other insets (right panels) show, respectively, the experimental geometry and the quarter-wave plate which varies the radiation helicity according to $P_{\text {circ }}=\sin 2 \varphi$. Full lines are fits to the phenomenological theory for $C_{2 v}$ symmetry relevant for (001)-grown unstructured III-V QWs and given by Eq. (34); see Ref. 19.

Radiation was applied at oblique incidence described by the angle of incidence $\theta_{0}$ varying from $-25^{\circ}$ to $+25^{\circ}$ (Fig. 2) and at normal incidence (Fig. 3). The current generated by THz light in the unbiased samples was measured via the voltage drop across a $50-\Omega$ load resistor in a closed-circuit configuration. The voltage was recorded with a storage oscilloscope.

\section{EXPERIMENTAL RESULTS}

We begin by introducing the results obtained from the reference samples. In the (001)-oriented unpatterned samples $\mathrm{R} 1$, as well as for R2 with symmetric groves, a signal is only detectable under oblique incidence. The photocurrent measured perpendicularly to the wave vector of the incident light is almost proportional to the helicity $P_{\text {circ }}$ and reverses its direction when the polarization switches from left- to right-handed circular (see the inset panel of Fig. 2). In the whole temperature range from room temperature to $4.2 \mathrm{~K}$ the variation of the angle of incidence from $\theta_{0}$ to $-\theta_{0}$ changes the sign of the photocurrent $J$. For normal incidence, the photocurrent vanishes. This is shown in Fig. 2 where the circular photocurrent in $\mathrm{R} 1$ is obtained by taking the difference between photoresponses to right- and left-handed radiation yielding the CPGE current $J_{C}=\left[J\left(\varphi=45^{\circ}\right)-J\left(\varphi=135^{\circ}\right)\right] / 2$. Similar results are obtained for the sample R2 (not shown). The $\theta_{0}$ and polarization dependencies of the photocurrent are in a good agreement with the phenomenological theory for the

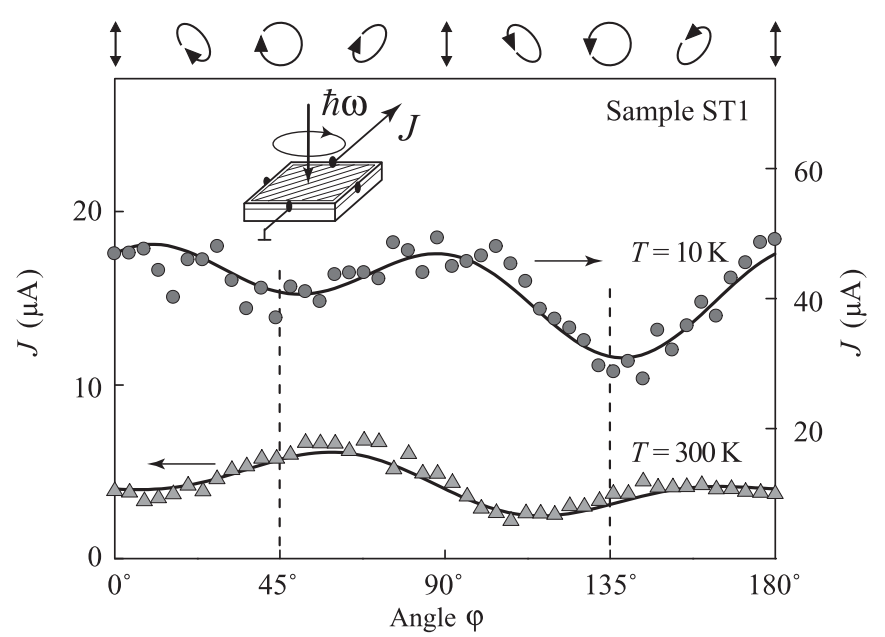

FIG. 3. Photocurrent measured as a function of the angle $\varphi$ at normal incidence $\left(\theta_{0}=0^{\circ}\right)$ in sample ST1 with the asymmetric lateral structure prepared along the [100] cubic axis. The current is measured at room temperature and $T=10 \mathrm{~K}$, excited by radiation with the wavelength $\lambda=280 \mu \mathrm{m}$ and power $P \approx 2 \mathrm{~kW}$. Full lines are fits to Eq. (35), see also Eq. (29). The inset shows the experimental geometry. The ellipses on top illustrate the state of polarization for various angles $\varphi$.

circular and linear photogalvanic effects obtained for the point group $C_{2 v}$. The total current is well fitted by Eqs. (14) describing the dominating circular photogalvanic current $J_{\text {ref }}$ of the unpatterned reference samples R1 and R2 as

$$
J_{\text {ref }}=J_{C \text { ref }} \sin \theta_{0} \xi P_{\text {circ }},
$$

where $J_{0, \text { ref }}=\gamma_{x^{\prime} y^{\prime}} t_{0}^{2} I_{0}, I_{0}$ is the incident intensity, $\xi=$ $t_{p} t_{s} / t_{0}^{2}, t_{p}$ and $t_{s}$ are the Fresnel transmission coefficients for $p$-and $s$-polarized light, respectively, and $t_{0}$ is the transmission coefficient for normal incidence. The corresponding fit of the circular photogalvanic current $J_{\text {ref }}$ excited by the right circularly polarized light $\left(P_{\text {circ }}=1\right)$ is shown by the full line in Fig. 2. A photocurrent, but with substantially smaller magnitude, is also obtained by applying linearly polarized radiation. This current is caused by the LPGE and its polarization behavior (not shown) is also well described by Eqs. (14).

The situation changes drastically for samples ST1 with asymmetric grating. Now a photocurrent can be detected even at normal incidence. The width of the observed photocurrent pulses is about $100 \mathrm{~ns}$, which corresponds to the duration of the $\mathrm{THz}$ laser pulse. In the patterned samples ST1 where the grooves are oriented along the [100] direction we have measured a magnitude of the photocurrent at normal incidence (Fig. 3) which is comparable and even larger than the one obtained in the reference sample $\mathrm{R} 1$ at large angles of incidence (Fig. 2). Moreover, the polarization behavior has changed. Figure 3 shows the photocurrent generated at normal incidence in sample ST1 as a function of the angle $\varphi$ indicating the helicity. The current is measured at an angle of $45^{\circ}$ with respect to the axes $x$ and $y$ and can be well fitted by an equal superposition of $j_{x}$ and $j_{y}$ of Eqs. (29) yielding

$$
J=J_{1}+J_{2} \mathcal{S}(\varphi)+J_{3} \mathcal{C}(\varphi)+J_{C} P_{\text {circ }}(\varphi) .
$$




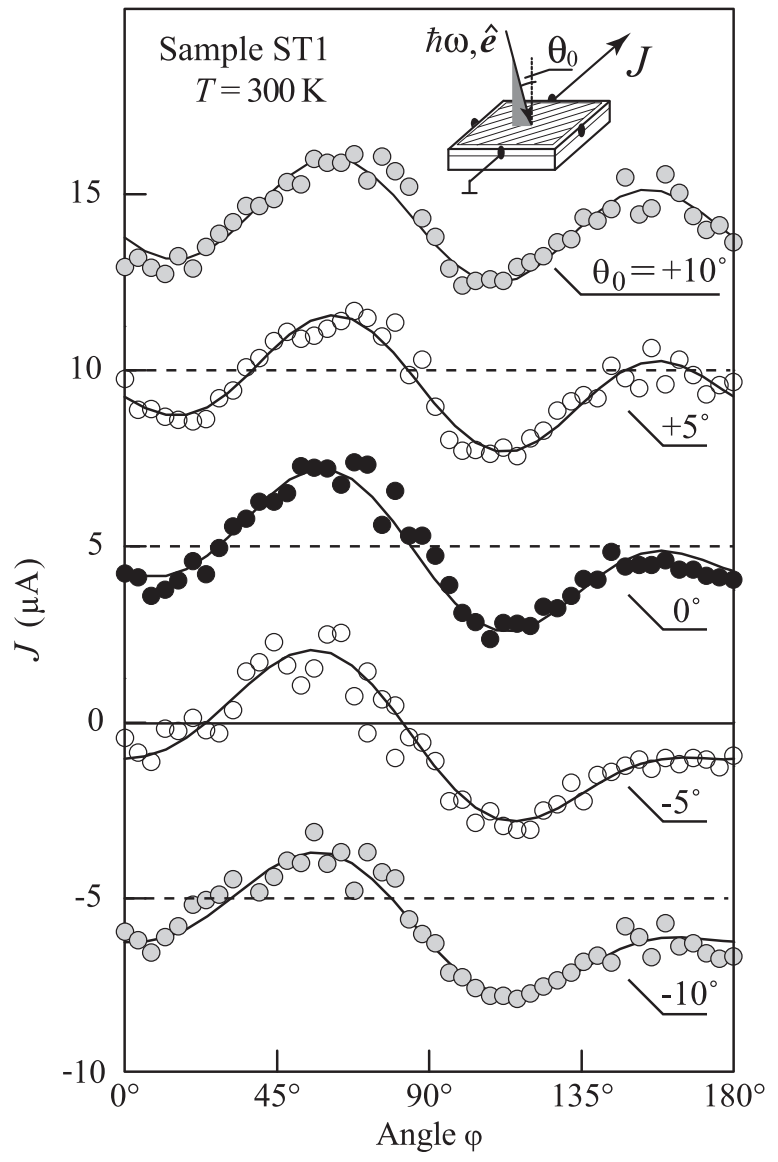

FIG. 4. Photocurrent measured as a function of the angle $\varphi$ at various angles of incidence $\theta_{0}$ in sample ST1 with an asymmetric lateral structure prepared along the [100] cubic axis. The data for $\theta_{0} \neq 0$ are shifted by $\pm 5 \mu \mathrm{A}$ for each $\pm 5^{\circ}$ step in $\theta_{0}$. The current is measured at room temperature, excited by radiation with the wavelength $\lambda$ equals; $280 \mu \mathrm{m}$ and power $P \approx 2 \mathrm{~kW}$. Full lines are fits to Eq. (35) [see also Eq. (29)]. The inset shows the experimental geometry.

Here, the fitting parameters $J_{j}(j=1,2,3)$ and $J_{C}$ are related to the coefficients $\chi_{j}$ and $-\gamma$ in Eq. (29) by the factor $\bar{I} / \sqrt{2}$. Figure 3 shows that the helicity dependent photocurrent $J_{C} P_{\text {circ }}(\varphi)$, denoted as circular transverse ratchet effect, contributes a substantial fraction to the total current.

While the normal-incidence photocurrent is observed at high and low temperatures and is always well described by the phenomenological Eq. (13) (see Fig. 3), the microscopic theory presented above [see Eqs. (29)-(31)] is only applicable for the room-temperature data. Indeed, the theory has been developed for the nondegenerate Boltzmann statistics and is invalid for the low-temperature region characterized by the Fermi energy $\varepsilon_{F} \gg k_{B} T$. What is even more important, with decreasing temperature the electron mean free path length $l_{e}$ becomes comparable with and even longer than the lateral superlattice period. This should be accompanied by a changeover from the mechanism related to the in-plane light intensity modulation with $j \propto \bar{I} h_{1} V_{1}$ to the mechanism of photocurrents arising in an asymmetric lateral potential for a homogeneous excitation and obtained by solving the kinetic equation (1) in the fifth-order perturbation theory, namely, the second order in the amplitude of the light electric field $E_{0}$ and the third order in the lateral potential; see Ref. 10. The measurement of the detailed temperature dependence of the photocurrent, the development of the theory in the case $l_{e}>d$, and the comparison between theory and experiment is beyond the scope of the present work. Therefore we focus below on the room-temperature data.

Equations (29) suggest that the ratchet currents display a maximum at normal incidence and are an even function with respect to the angle of incidence. In order to verify this we measured the polarization dependence of the photocurrent for various angles of incidence. The corresponding data are shown in Fig. 4. In this figure the full lines are fits to Eq. (35), which is applicable not only for normal but also for oblique incidence. In the latter case, the current is a sum of contributions due to the ratchet effect described by Eqs. (29) and the photogalvanic effect given by Eqs. (14). Figure 5 displays the magnitude of the helicity dependent currents as a function of the angle of incidence $\theta_{0}$ for sample ST1 (full circles) and the unstructured reference sample R1 (open circles). In addition, the $\theta_{0}$ dependence of the other three contributions in Eq. (35) are shown for the sample ST1. To extract the current $J_{\text {circ }}$ from the total current we used the fact that the corresponding contribution to $J$ is proportional to $\sin 2 \varphi$ and changes its sign upon switching the helicity while all the other terms remain unchanged. Taking the difference of photocurrents of right- and left-handed radiation, we get the values of $J_{\text {circ }}$.

As addressed above, in the structured sample ST1, the current $J_{\text {circ }}$ excited at oblique incidence consists of two contributions. The first one has the same origin as the one observed in the reference sample R1 and is described by Eq. (34). The second one is due to the lateral structure and corresponds to the transverse ratchet effect. The dependence of the circular photocurrent on the angle of incidence $\theta_{0}$ can be well fitted by

$$
J_{\text {circ }}=\left(J_{0, \text { ref }} \sin \theta_{0}+J_{C} \cos \theta_{0}\right) \xi .
$$

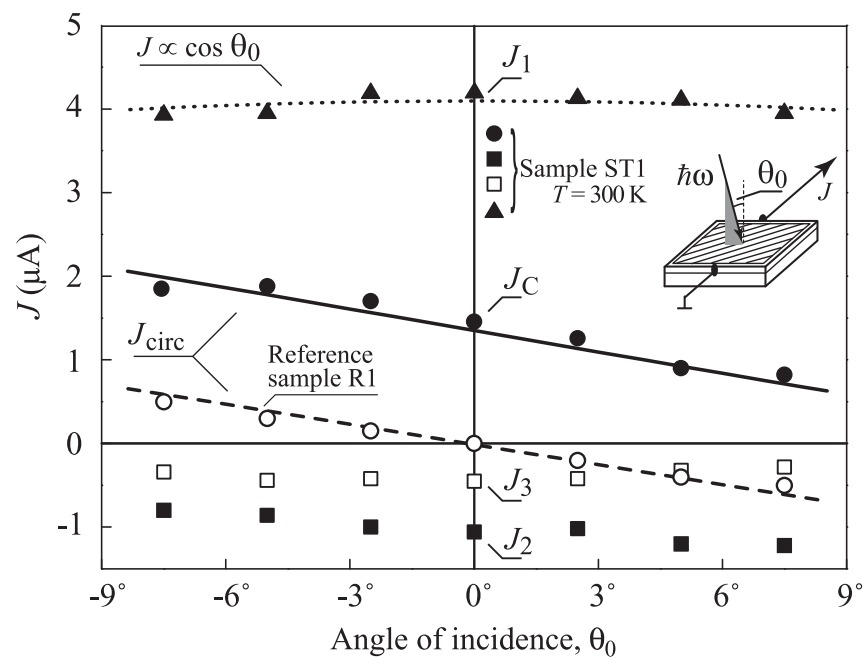

FIG. 5. Angle of incidence dependence of the photocurrent. - and $\circ$ : current $J_{\text {circ }}$ measured in sample ST1 and the reference sample R1, respectively. $\square, \boldsymbol{\square}$, and $\boldsymbol{\Delta}$ are current contributions proportional to $J_{3}, J_{2}$, and $J_{1}$. Dotted line is the fit after Eq. (38), solid and dashed lines to Eq. (36). 


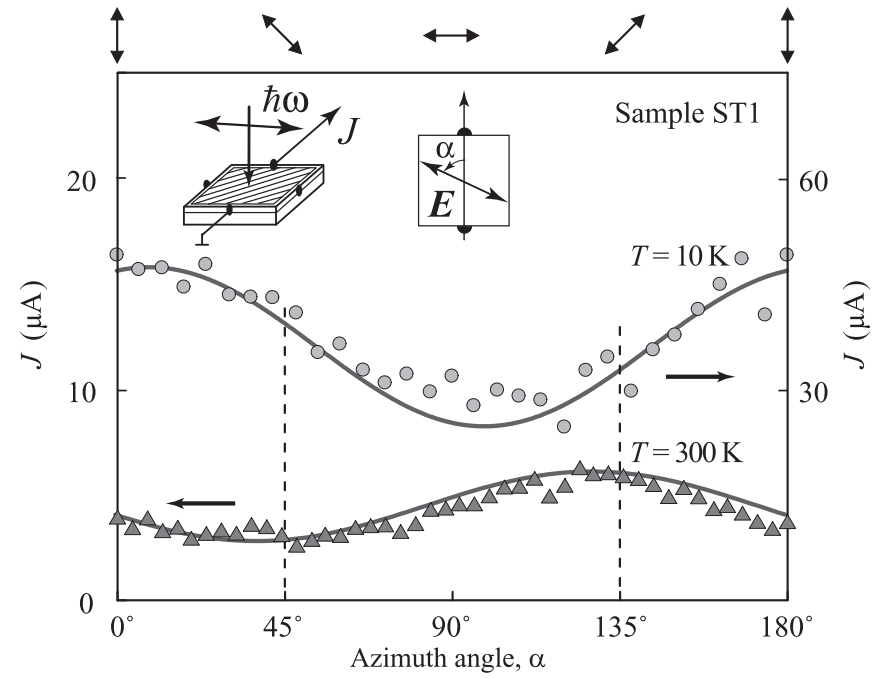

FIG. 6. Photocurrent $J$ measured as a function of the azimuth angle $\alpha$ under normal incidence at room temperature and $T=10 \mathrm{~K}$ in sample ST1 with the asymmetric lateral structure along the [100] axis. The photocurrent is excited by linearly polarized radiation with the wavelength $\lambda=280 \mu \mathrm{m}$ and power $P \approx 2 \mathrm{~kW}$. Full lines are fits to Eq. (37); see also Eq. (29). We used for fitting the same values of $J_{j}$ as in the experiments with elliptically polarized radiation; see Fig. 3. Left inset shows the experimental geometry, and right inset defines the angle $\alpha$. Arrows on top indicate the polarization corresponding to various values of $\alpha$.

Now we turn to the photon helicity independent contributions to the photocurrent, denoted by the coefficients $J_{j}$ in Eq. (35), which describe photocurrents generated by linearly polarized radiation and a polarization independent contribution $J_{1}$. Figure 6 shows the dependence of the normal-incidence photocurrent $J$ on the azimuth angle $\alpha$. We have found that all data can be well fitted by

$$
J=J_{1}+J_{2} \mathcal{S}(\alpha)+J_{3} \mathcal{C}(\alpha)
$$

in agreement with the microscopic theory of ratchet effects given by Eqs. (29). We emphasize that, as expected from the theory, $J_{j}$ are the same fitting parameters as the ones used for the data shown in Fig. 3. Figure 5 shows the dependence of the polarization independent contribution, proportional to the coefficient $J_{1}$, on the angle of incidence $\theta_{0}$. In this case, the experimental data can be well fitted by

$$
J=J_{1} \cos \theta_{0} \xi .
$$

Figures 3 and 6 demonstrate that the dominant contribution to the photocurrent is polarization independent and can therefore be obtained by unpolarized radiation. In the microscopic theory this photocurrent is mostly due to Seebeck ratchet effect and is described by the term proportional to $\chi_{1}$ in Eqs. (29).

The results obtained on the second set of lateral samples, ST2, are shown in Fig. 7. Again, we start the discussion with the photocurrent in the reference, here the unstructured part of the Hall bar (see inset of Fig. 7). Also here, a photocurrent is only observed at oblique incidence. This finding is in agreement with the results above underlining once again that ratchet effects do not occur in unpatterned structures. In the patterned part of the sample, however, a remarkable

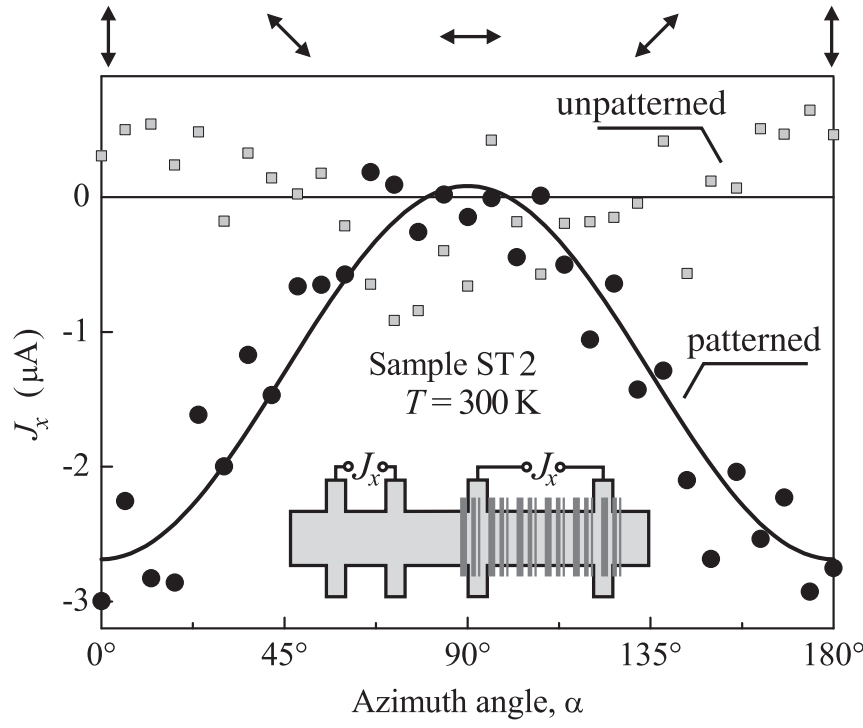

FIG. 7. Photocurrent $J_{x}$ measured at sample ST2 as a function of the azimuth angle $\alpha$ at normal incidence and a wavelength of $\lambda=280 \mu \mathrm{m}$ and power $P \approx 9 \mathrm{~kW}$. The dependences for both parts with and without asymmetric stripes are shown. The current induced in the structured part by linearly polarized radiation is well fitted by Eq. (39); see also Eq. (29). The inset displays the design of the Hall bar with the structured and the unpatterned part. Arrows on top indicate the polarization corresponding to various values of $\alpha$.

photocurrent $J_{x}$ can be observed at normal incidence. As shown in Fig. 7, the current, which flows perpendicularly to the asymmetric stripes, strongly depends on the azimuth angle $\alpha$ of the light's polarization defined above and can be well fitted by

$$
J=J_{1}+J_{2} \mathcal{C}(\alpha) .
$$

This is fully in line with the theory of the ratchet effect discussed above (see Secs. III and VI) and given by Eqs. (29). Our observation demonstrates that an asymmetric periodic potential can be controllably introduced by the $A B C$ gate. We note that in this sample the interplay of the polarization independent Seebeck photocurrent $J_{1}$ [Eq. (24)] and linear ratchet photocurrent proportional to $J_{2}$ [or to the coefficient

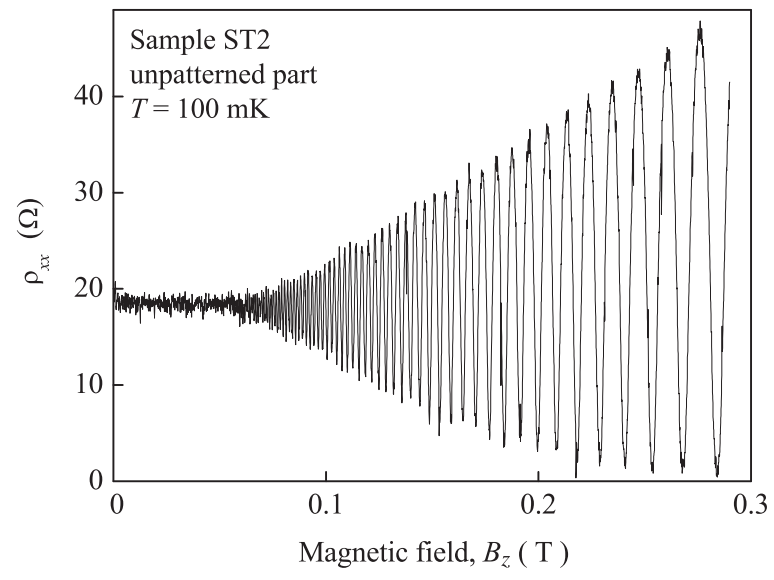

FIG. 8. Shubnikov-de Haas oscillations measured at $100 \mathrm{mK}$ in the unpatterned reference section of the ST2 sample. 


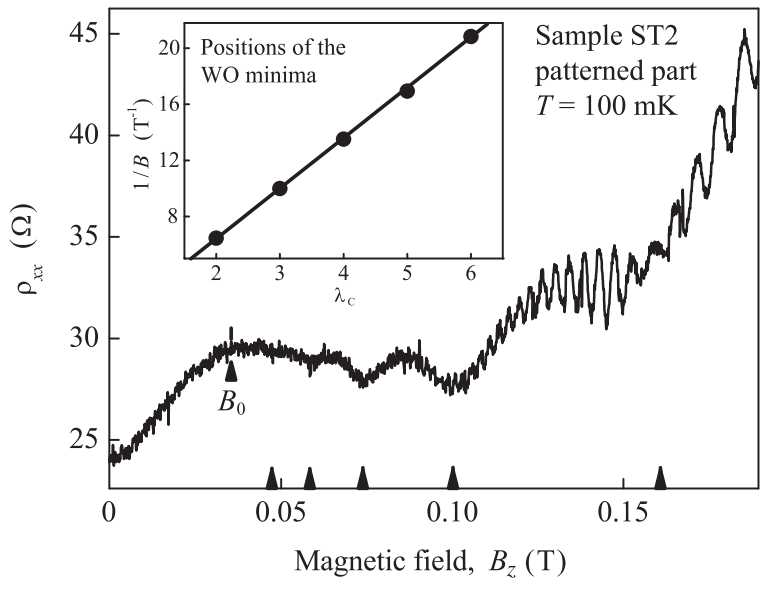

FIG. 9. Longitudinal resistance $\rho_{x x}$ in the modulated part of the Hall bar sample ST2. At low $B, 1 / B$ periodic commensurability oscillation indicate the presence of a weak periodic potential. The $1 / B$ periodicity of the low-field oscillations is evident from the inset where the oscillation index $\lambda_{C}$ is plotted vs the resistance minima position $1 / B$.

$\chi_{2}$ in Eqs. (29)] yields a maximum of the signal for the radiation electric field aligned perpendicular to the direction of modulation.

To check that the 2DES potential is in fact modulated, we carried out magnetotransport measurements at low temperatures. Corresponding data of the longitudinal resistance $\rho_{x x}$ of the unpatterned reference area as a function of the magnetic field $B_{z}$ are shown in Fig. 8 and display pronounced Shubnikov-de Haas oscillations. As the mean free path $l_{e}$ in the superlattice device is about $9 \mu \mathrm{m}$ and hence longer than the period of the SL as well as much longer than the average distance between neighboring finger strips we expect commensurability effects to occur. ${ }^{27}$ In this limit the periodic potential causes $1 / B$ periodic resistance oscillation where minima are given by the condition

$$
2 R_{C}=\left(\lambda_{C}-\frac{1}{4}\right) d, \quad \lambda_{C}=1,2,3 \cdots .
$$

Here, $2 R_{C}$ is the semiclassical cyclotron orbit diameter and $\lambda_{C}$ is the oscillation index. Such commensurability (or Weiss oscillations) are clearly visible at low magnetic fields of the trace measured in the superlattice part of the sample, as presented in Fig. 9. The SL period $d$ extracted from the Weiss oscillations (WO) is about $570 \mathrm{~nm}$ and agrees with one of the Fourier components of the asymmetric periodic potential.
Why this particular Fourier component dominates is a subject of future investigations. Qualitatively it is understandable that the contribution stemming from the full periodicity of $3.8 \mu \mathrm{m}$ is cut off due to scattering, as the circumference of the corresponding cyclotron orbit is about $l_{e}$, and that contributions with much smaller periodicity are cut off as the corresponding Fourier coefficients get exponentially damped with increasing surface-2DES distance. ${ }^{28}$ Nonetheless, the presence of commensurability effects is a clear signature of the presence of a weak periodic potential.

\section{SUMMARY}

The lateral grating etched into the sample's surface or deposition of periodic metal stripes on the sample top induce a periodic lateral potential acting on the two-dimensional electron gas. As a consequence, the magnetotransport properties of the heterostructure changes and $1 / B$ oscillations appear at low temperatures in the longitudinal magnetoresistance. In addition, if illuminated, it modifies the normally incident radiation causing its spatial modulation in plane of the electron gas. If the lateral superlattice is asymmetric the spatial modulations of the static lateral potential $V(x)$ and the radiation intensity $I(x)$ are shifted relative to each other. As a result the product of the static force $-d V(x) / d x$ and the photothermal modulation of the electron density $\delta N(x)$ has a nonzero space average and therefore a homogeneous electric current is generated, an effect previously predicted by Blanter and Büttiker. ${ }^{15}$ The class of electronic ratchets is extended to polarization-sensitive linear and circular ratchets. The ratchet currents which are sensitive to the linear and circular polarization of the light arise in the same system with broken symmetry due to the phase shift between the periodic potential and the periodic light field resulting from near field diffraction. They appear because the carriers in the laterally modulated quantum wells move in two directions and are subjected to the action of the two-component electric field. In contrast to the photothermal current, the linear and circular ratchet currents are independent of the energy relaxation time and controlled only by the momentum relaxation time.

\section{ACKNOWLEDGMENTS}

Support from DFG (SFB 689), Linkage Grant of IB of BMBF at DLR, RFBR, and the Russian Ministry of Education and Sciences is gratefully acknowledged. We are grateful to M. Grifoni, M. Büttiker, and V. V. Bel'kov for fruitful discussions, as well as C. Linz and R. Ravash for sample preparation. *sergey.ganichev@physik.uni-regensburg.de

${ }^{1}$ P. Reimann, Phys. Rep. 361, 57 (2002).

${ }^{2}$ Ratchets and Brownian Motors: Basic Experiments and Applications, edited by H. Linke, special issue Appl. Phys. A: Mater. Sci. Process. A 75, 167 (2002).

${ }^{3}$ P. Hänggi, F. Marchesoni, and F. Nori, Ann. Phys. (Leipzig) 14, 51 (2005).
${ }^{4}$ P. Reimann, M. Grifoni, and P. Hänggi, Phys. Rev. Lett. 79, 10 (1997).

${ }^{5}$ A. Lorke, S. Wimmer, B. Jager, J. P. Kotthaus, W. Wegscheider, and M. Bichler, Physica B (Amsterdam) 249-251, 312 (1998).

${ }^{6}$ H. Linke, T. E. Humphrey, A. Lofgren, A. O. Sushkov, R. Newbury, R. P. Taylor, and P. Omling, Science 286, 2314 (1999). 
${ }^{7}$ A. M. Song, P. Omling, L. Samuelson, W. Seifert, I. Shorubalko, and H. Zirath, Appl. Phys. Lett. 79, 1357 (2001).

${ }^{8}$ J. B. Majer, J. Peguiron, M. Grifoni, M. Tusveld, and J. E. Mooij, Phys. Rev. Lett. 90, 056802 (2003).

${ }^{9}$ M. V. Costache and S. O. Valenzuela, Science 330, 1645 (2010).

${ }^{10}$ L. I. Magarill, Physica E (Amsterdam) 9, 652 (2001).

${ }^{11}$ M. V. Entin and L. I. Magarill, Phys. Rev. B 73, 205206 (2006).

${ }^{12}$ A. D. Chepelianskii, Eur. Phys. J. B 52, 389 (2006).

${ }^{13}$ A. D. Chepelianskii, M. V. Entin, L. I. Magarill, and D. L. Chepelyansky, Eur. Phys. J. B 56, 323 (2007); Physica E (Amsterdam) 40, 1264 (2008).

${ }^{14}$ W. Weber, L. E. Golub, S. N. Danilov, J. Karch, C. Reitmaier, B. Wittmann, V. V. Bel'kov, E. L. Ivchenko, Z. D. Kvon, N. Q. Vinh, A. F. G. van der Meer, B. Murdin, and S. D. Ganichev, Phys. Rev. B 77, 245304 (2008).

${ }^{15}$ Ya. M. Blanter and M. Büttiker, Phys. Rev. Lett. 81, 4040 (1998).

${ }^{16}$ P. Olbrich, E. L. Ivchenko, R. Ravash, T. Feil, S. D. Danilov, J. Allerdings, D. Weiss, D. Schuh, W. Wegscheider, and S. D. Ganichev, Phys. Rev. Lett. 103, 090603 (2009).
${ }^{17}$ M. Büttiker, Z. Phys. B 68, 161 (1987).

${ }^{18}$ S. D. Ganichev, E. L. Ivchenko, and W. Prettl, Physica E (Amsterdam) 14, 166 (2002).

${ }^{19}$ E. L. Ivchenko, Optical Spectroscopy of Semiconductor Nanostructures (Alpha Science International, Harrow, UK, 2005).

${ }^{20}$ F. G. Bass and Yu. G. Gurevich, Sov. Phys. Usp. 14, 113 (1971).

${ }^{21}$ S. D. Ganichev, E. L. Ivchenko, R. Ya. Rasulov, I. D. Yaroshetskii, and B. Ya. Averbuch, Phys. Solid State 35, 104 (1993).

${ }^{22}$ E. L. Ivchenko and B. Spivak, Phys. Rev. B 66, 155404 (2002).

${ }^{23}$ S. Adachi and K. Oe, J. Electrochem. Soc. 130, 2427 (1983).

${ }^{24}$ S. Adachi, Jpn. J. Appl. Phys. 30, 1196 (1991).

${ }^{25} \mathrm{~S}$. D. Ganichev and W. Prettl, Intense Terahertz Excitation of Semiconductors (Oxford University Press, Oxford, 2006).

${ }^{26}$ S. D. Ganichev, S. A. Emel'yanov, and I. D. Yaroshetskii, Pis'ma Zh. Eksp. Teor. Fiz. 35, 297 (1982) [JETP Lett. 35, 368 (1982)].

${ }^{27}$ D. Weiss, K. v. Klitzing, K. Ploog, and G. Weimann, Europhys. Lett. 8, 179 (1989).

${ }^{28}$ R. R. Gerhardts, D. Weiss, and K. v. Klitzing, Phys. Rev. Lett. 62, 1173 (1989). 